\title{
Percepción de Riesgo de Exposición Ocupacional a Sílice: Construcción y Valídación de un Cuestionario en Chile
}

\author{
RISK PERCEPTION OF OCCUPATIONAL EXPOSURE TO SILICA: CONSTRUCTION AND VALIDATION OF A \\ QUESTIONNAIRE IN CHILE
}

\author{
Ximena Ossa', Olga Vásquez ${ }^{2}$, Jossiana Robinovich ${ }^{3}$, Luis Bustos ${ }^{4}$, Juan José Orellana ${ }^{5}$ \\ 1. Doctor en Salud Pública, Departamento de Salud Pública y CIGES, Universidad de La Frontera, Temuco, Chile. \\ 2. Doctor en Ciencias Sociales, Escuela de Ciencias de la Salud, Universidad Católica de Temuco, Chile. \\ 3. Doctor ${ }^{\odot}$ en Salud Pública, Departamento de Salud Pública, Universidad de La Frontera, Temuco, Chile. \\ 4. Estadístico, Departamento de Salud Pública, Universidad de La Frontera, Temuco, Chile. \\ 5. Doctor en Salud Pública, Departamento de Salud Pública y CIGES, Universidad de La Frontera, Temuco, Chile.
}

\begin{abstract}
RESUMEN
Introducción: La silicosis es una enfermedad pulmonar progresiva, irreversible, pero potencialmente prevenible. Conocer la percepción de riesgo en trabajadores expuestos a sílice es necesario para intervenir y reducir la incidencia de silicosis y otras enfermedades relacionadas. El objetivo de este estudio fue validar un Cuestionario de Percepción de Riesgo de Exposición Ocupacional a Sílice (CuPREOS) en trabajadores expuestos, en distintas actividades laborales en Temuco, Chile. Material y método: Una etapa de exploración con enfoque fenomenológico/ naturalista permitió conformar categorias de primer y segundo orden, que concluyó en una primera versión del instrumento. Una segunda etapa incluyó análisis factorial exploratorio y confirmatorio. Resultados: Se develaron dos dimensiones subyacentes: a) evidencia de riesgo, en la que el trabajador manifiesta evaluar señales de alarma y b) ausencia de riesgo, en la que sopesa aspectos que contrarrestan las anteriores. Ambas configuran la estructura conceptual que conforma el CuPREOS de 9 items/preguntas, con una confiabilidad de 0.84 (alfa Crönbach). Los indicadores del análisis confirmatorio revelan un buen ajuste del modelo. Discusión: la percepción de riesgo es un constructo que involucra complejidades sociales y culturales, su medición debe ser sencilla y práctica; su conocimiento permitiría decisiones más efectivas en prevención e intervención.
\end{abstract}

(Ossa X, Vásquez 0, RobinovichJ, Bustos L, Orellana J, 2018. Percepción de Riesgo de Exposición Ocupacional a Sílice: Construcción y Validación de un Cuestionario en Chile. Cienc Trab. May-Ago; 20 [62]: 90-96).

Palabras claves: ESTUDIOS DE VALIDACIÓN, SÍLICE LIBRE, SILICOSIS, PERCEPCIÓN, RIESGOS LABORALES.

\section{ABSTRACT}

Introduction: Silicosis is a progressive lung disease, irreversible, but potentially preventable. Knowing the perception of risk in workers exposed to silica is necessary to intervene and reduce the incidence of silicosis and other related diseases. The objective of this study was to validate a Questionnaire of risk perception of occupational exposure to silica (CUPREOS) in exposed workers, in different work activities in Temuco, Chile. Material and method: A stage of exploration with phenomenological/naturalist approach allowed to create categories of first and second order, which ended in a first version of the instrument. The second stage included exploratory and confirmatory factor analysis. Results: two underlying dimensions were revealed: a) evidence of risk, in which worker states to evaluate signals of alarm and b) absence of risk, in which he weighs aspects that counteract the previous ones. Both set up the conceptual framework that sustain the CuPREOS of 9 items/questions, with a reliability of 0.84 (Cronbach alfa). The confirmatory analysis indicators show a good fit of the model. Discussion: the perception of risk is a construct involving social and cultural complexities, its measurement should be simple and practical; its knowledge would enable more effective decision-making in prevention and intervention.

Key words: VALIDATION STUDIES, FREE SILICA, SILICOSIS, PERCEPTION, OCCUPATIONAL RISK.

\section{INTRODUCCIÓN}

La silicosis, enfermedad pulmonar progresiva e irreversible de origen ocupacional, es potencialmente prevenible. Es producida como consecuencia de la inhalación sostenida de polvo con alto

\section{Correspondencia / Correspondence:}

Dra. Ximena Ossa

Gral. Carrera 228, Temuco, Chile.

Tel.: 56-452592185

e-mail: ximena.ossa@ufrontera.cl

Recibido: 18 de Enero de 2018 / Aceptado: 25 de Julio de 2018 contenido de sílice libre cristalizada en fracción respirable al desarrollar trabajos relacionados con materiales provenientes de la corteza terrestre.

La progresión del compromiso funcional pulmonar, así como el periodo de latencia antes de la manifestación clínica se correlacionan con la intensidad y el tiempo de exposición en la actividad laboral, pudiendo causar finalmente invalidez física y muerte. ${ }^{1} \mathrm{La}$ evidencia muestra que también existe asociación entre exposición a sílice y cáncer de pulmón, enfermedad pulmonar obstructiva crónica, tuberculosis pulmonar y artritis reumatoide, entre otras. ${ }^{2.3}$ Durante bastante tiempo la exposición a sílice se relacionaba con la labor minera; actualmente, se conoce que otras actividades laborales también tienen un alto riesgo de exposición. Entre estas 
se encuentran la fabricación de ladrillos, enlozados, baldosas, cerámicas, plantas de áridos, fundiciones de hierro, molienda de cuarzo, corte, pulido y grabado de vidrios, mármol y actividades del rubro de la construcción, especialmente el desbaste de cemento. ${ }^{4}$ Por su relevancia para minimizar o evitar el daño a la salud, la mayor parte de los estudios sobre este tema se enfocan en la cuantificación del riesgo de exposición y en la efectividad de la implementación y uso de medidas preventivas. ${ }^{4-7}$ Estos estudios muestran que la utilización de dispositivos de protección no depende exclusivamente de que existan estos en el lugar de trabajo, sino también de factores individuales y de la cultura laboral existente. ${ }^{8-10}$ Un aspecto relevante y poco estudiado es la percepción del riesgo al que se está expuesto, lo que está condicionado, entre otros factores, por el conocimiento de los efectos de la exposición y la cultura del lugar de trabajo en relación con la protección. ${ }^{8,11,12}$

Existe evidencia que en trabajos con explícitos peligros para la salud se observa un sesgo optimista entre los trabajadores en relación con su autopercepción de riesgo. Estos consideran que la propia exposición al riesgo es menor que el promedio de sus compañeros, y que ellos son capaces de mantenerse a salvo en sus lugares de trabajo. ${ }^{13,14}$ Las personas saludables tienden a quedarse con la información más deseable o ventajosa para ajustar sus creencias con respecto a su propio riesgo, lo cual puede tener costos importantes para su salud cuando están expuestos a un peligro. ${ }^{15}$

Según algunos autores, la percepción del riesgo es construida socialmente y sesgada por el ambiente o cultura donde se desenvuelve el individuo. No es una cuestión individual sino más bien una visión intersubjetiva que involucra conocimientos y creencias compartidas, que justifican diferentes formas de comportamiento. ${ }^{16}$ La evidencia sugiere una asociación directamente proporcional entre percepción de riesgo y comportamiento preventivo individual en diversas áreas relacionadas con salud (exposición a asbesto, radiación solar y ruido ambiental, entre otros); ${ }^{8,17-20}$ sin embargo, la investigación en trabajadores expuestos a sílice escasamente indaga sobre percepción de riesgo.

En varios países de todos los continentes se ha develado que la exposición a sílice en trabajos distintos de la minería ha aumentado en la medida que la industrialización y comercialización de nuevos productos y artículos con contenido de sílice se han diversificado. ${ }^{21-26}$ Por otro lado, la crisis económica global ha puesto una voz de alarma en relación con la dificultad de monitorear la ocurrencia de enfermedades causadas por exposiciones riesgosas debido a despidos masivos de industrias formales, con la consiguiente posibilidad de instalación de trabajo informal. ${ }^{27}$ Actualmente, en Chile se estima que 5.4\% de la fuerza de trabajo ocupada, de diversos rubros, tiene alta probabilidad de exposición a este compuesto ${ }^{4}$, razón por lo cual esta situación se considera un problema prioritario de salud ocupacional chilena. En 2009, se implementó el "Plan Nacional de Erradicación de la Silicosis" (PLANESI), cuyo objetivo es erradicar esta enfermedad en el país para el año 2030, siguiendo los lineamientos emanados de la Organización Mundial de la Salud y la Organización Internacional del Trabajo. ${ }^{28}$

Es necesario considerar que muchos trabajadores que diariamente se exponen a sílice durante su jornada laboral trabajan en pequeñas empresas unipersonales o familiares, en calidad de informales ${ }^{29}$, con deficiente gestión y prevención de riesgos. Esto resalta la necesidad de evaluar los niveles de percepción de riesgo en trabajadores tanto formales como informales, ya que de ello puede depender el compromiso con un comportamiento preven- tivo. De acuerdo a los argumentos anteriores, el objetivo de este estudio fue desarrollar y validar un cuestionario de percepción de riesgo de exposición ocupacional a sílice (CUPREOS) en trabajadores expuestos de la Región de La Araucanía, Chile. Esta región no minera se caracteriza por presentar los indicadores socioeconómicos más bajos del país, y estar ubicada en la zona con mayor nivel de emprendimiento laboral informal, especialmente en rubros relacionados con exposición a sílice como la construcción y algunas manufacturas. ${ }^{29}$

\section{MATERIAL Y MÉTODO}

CUPREOS fue desarrollado y validado utilizando un método mixto en dos etapas. La primera fue una exploración cualitativa con un enfoque fenomenológico/naturalista para comprender el proceso cognitivo, significados y representaciones que le adjudican los trabajadores al riesgo de exposición a sílice. ${ }^{30}$ La segunda etapa correspondió a una fase cuantitativa de validación mediante análisis psicométrico y evaluación de la confiabilidad del instrumento. ${ }^{31}$

\section{Primera etapa: Construcción del instrumento}

Inicialmente, se realizó una búsqueda sistemática de literatura para identificar instrumentos similares en estudios nacionales e internacionales y literatura ad-hoc. Posteriormente, se llevó a cabo el trabajo de campo cualitativo, identificando informantes claves: prevencionistas de riesgo con experiencia en vigilancia y fiscalización de normativa vigente relacionada con exposición a sílice y trabajadores de diferentes rubros, potencialmente expuestos a sílice. Se realizaron grupos focales tanto de expertos como de trabajadores $\mathrm{y}$, posteriormente, se entrevistó en profundidad a algunos de los trabajadores para indagar en profundidad aspectos que emergieron de la dinámica focal, hasta saturar datos. La pregunta guía con la cual se trabajó fue: ¿cómo se da cuenta o percibe que su salud está en riesgo o peligro cuando está expuesto al polvo que se produce mientras trabaja? Todos los participantes accedieron a firmar un formulario de consentimiento informado. El estudio fue aprobado por el Comité Ético Científico de la Universidad de La Frontera, Temuco, Chile.

Los relatos fueron audiograbados y luego transcritos rigurosamente. Los datos fueron reducidos a contenidos esenciales, los cuales se analizaron según análisis convencional de contenidos cualitativos. ${ }^{32}$ Los datos se codificaron en forma exhaustiva identificando las dimensiones principales (categorías de primer orden), y en cada una de ellas indicadores (categorías de segundo orden), a partir de los cuales se originaron los ítems del cuestionario. Este proceso fue realizado con ayuda del paquete computacional Atlas Ti.

Posteriormente, se construyeron preguntas asociadas a cada uno de los items, las que fueron sometidas a discusión y análisis; se eliminaron algunas por problemas de lenguaje, inconsistencias y relación tangencial con la temática central, dando lugar a la primera versión del CuPREOS, con un total de 13 items/preguntas.

\section{Segunda etapa: Validación y confiabilidad del instrumento}

La primera versión del instrumento fue sometida a validez de fachada, aplicando la técnica de Juicio de Expertos: un experto en riesgo con amplia experiencia en el área, un ingeniero químico que comparte autoría del PLANESI y un investigador del área de salud laboral relacionada a contaminación por sílice, evaluaron el instrumento utilizando una pauta para analizar cada pregunta en relación 
con relevancia, pertinencia, comprensión y sensibilidad de la escala de respuesta utilizada. El objetivo fue obtener la opinión de cada uno respecto de las características de calidad aparente del cuestionario. ${ }^{33}$ Incorporados los aportes, la segunda versión se sometió a validación de contenido aplicándola a 10 trabajadores de diferentes rubros con potencial exposición a polvo de sílice. El objetivo fue evaluar comprensión, representatividad y adecuación cultural de los items/ preguntas. Luego de realizadas las modificaciones aportadas por este proceso, se obtuvo la tercera versión del cuestionario. En una última etapa, para la validación de constructo, el instrumento fue aplicado a una muestra de 77 trabajadores que aceptaron participar del estudio, firmando un formulario de consentimiento informado, de un total de 100 trabajadores identificados que se desempeñaban en distintos rubros con potencial exposición a sílice. El tamaño de la muestra se consideró adecuado ya que supera el mínimo aceptable para realizar validación de constructo ( 3 participantes por ítem) ${ }^{34} \mathrm{y}$ porque las comunalidades encontradas $(>0.6)$ respaldaron este número como adecuado. ${ }^{35,36}$

A través de análisis factorial exploratorio (AFE) se realizó análisis descriptivo de los ítems, índice de Kayser_Meyer-Olkin, test de Barlett, extracción de comunalidades con método de rotación Varimax, cálculo de Alfa de Crönbach y correlación ítem-total para evaluar fiabilidad a través de la consistencia interna del instrumento. ${ }^{34,37}$ Posteriormente, se sometió a análisis confirmatorio a través de Análisis Generalizado de Componentes Estructurales (GSCA). El AFE se realizó con el paquete estadístico IBM Statistics SPSS 22; y para el GSCA, se utilizó el programa web de libre acceso GSCA (http://www.sem-gesca.org/).

\section{RESULTADOS}

\section{Primera etapa: Construcción del instrumento}

La búsqueda sistemática se realizó en las bases de datos de pubmed, Embase, ISI Web of Science, Scopus, Lilacs y Scielo, agregándose una búsqueda libre en Scholar Google. No se encontraron resultados efectivos.

Para la exploración cualitativa se invitó a 12 prevencionistas de riesgo y a 20 trabajadores a participar de los grupos focales. De los primeros, concurrieron 10 participantes, pero en el caso de los trabajadores solo asistieron ${ }^{4}$. Los motivos aludidos para no acudir a la invitación fueron falta de tiempo, de interés y olvido, entre otros. Las entrevistas en profundidad se realizaron a cinco trabajadores (quienes no participaron en el grupo focal) en sus lugares de trabajo (cortador de vidrios, laboratorista dental, yesero, albañil y marmolero).

El análisis de contenidos de estos datos cualitativos reveló tres dimensiones subyacentes: evidencia de riesgo, ausencia de riesgo y presencia de materiales peligrosos. Estas dimensiones, descritas en los siguientes párrafos, conforman el ámbito conceptual en el que se enmarca la percepción del riesgo de exposición a sílice. Aunque en apariencia son dependientes e interrelacionadas, las dimensiones identificadas revelan percepciones divergentes en relación con el riesgo de exposición entre los trabajadores.

\section{Dimensión Evidencia de riesgo}

Los indicadores que se mencionan en esta dimensión tienen relación con lo que motiva a los trabajadores a buscar o, al menos, a pensar en utilizar medidas de protección. Consideran signos de alarma inmediata la presencia en el aire de polvillo, gases tóxicos y otros mate- riales peligrosos (contaminación ambiental en general), sin embargo, para ellos, de estos tres, el primero es el menos importante. Asumen que la presencia de cualquiera de estos elementos significa riesgo para su salud, si hay más de uno la percepción de potencial daño es mayor. No diferencian claramente que es el polvo, en su fracción respirable, el que puede provocarles silicosis, sino que perciben como peligroso todo aquello potencialmente respirable. Si a estos factores se le suma una escasa ventilación, la percepción de riesgo es mayor; no obstante, esta última condición por sí sola también genera sensación de peligro, aunque no tan severo.

Los trabajadores y prevencionistas de riesgo tienen una percepción diferente con respecto a la disponibilidad de dispositivos de protección y de informativos de alerta de peligro, así como la presencia de estos profesionales en el campo laboral. Bajo la perspectiva de los prevencionistas, los ambientes laborales se tornan más seguros debido a las medidas de prevención descritas; en cambio, para la mayor parte de los trabajadores entrevistados, la presencia de elementos preventivos, incluyendo al prevencionista en el campo laboral, es una consecuencia de un riesgo elevado, y la cantidad y variedad de estas medidas es proporcional al riesgo al cual se exponen. Es decir, mientras más dispositivos y personal de seguridad existan, para ellos, el riesgo es mayor.

\section{Dimensión ausencia de riesgo}

En esta dimensión, los indicadores que emergen son elementos cuya presencia permite a los trabajadores catalogar el ambiente laboral como de escaso peligro para la salud. Su apreciación no siempre es correcta, y tampoco equivale necesariamente a lo opuesto o contrario de los indicadores que aportan a la dimensión evidencia de riesgo.

La humidificación de los procesos laborales que emiten polvo al ambiente, la presencia de ventilación espontánea (ventanas abiertas con corrientes de aire) o forzada (ventiladores/extractores) y los espacios muy abiertos provocan que los trabajadores perciban un riesgo de exposición a sílice muy bajo o nulo. La ausencia de polvillo visible en el ambiente de trabajo crea una sensación errónea de seguridad. La mayoría de los trabajadores encuestados no tiene conocimiento de que el material particulado fino, potencialmente respirable, cuyo pequeño diámetro ( $\leq 2,5 \mathrm{ppm})$ lo hace indistinguible o invisible al ojo humano es el que reviste mayor peligro para su salud. Bajo la perspectiva de los trabajadores, no hay riesgo si no se visualiza abundante polvo en suspensión en el ambiente. La decisión que según ellos corresponde tomar con respecto a los dispositivos de protección en esta circunstancia es disminuir su uso, o definitivamente no utilizarlos.

Por otra parte, los prevencionistas de riesgo hacen especial mención de los procedimientos de limpieza utilizados en el lugar de trabajo. Ellos afirman que los trabajadores no dan a este proceso la importancia que tiene con respecto a la generación de material particulado en suspensión. Esto es reafirmado por el relato de los aludidos, quienes no se refieren a los procedimientos de limpieza cuando se les pregunta sobre condiciones de riesgo de exposición a sílice en su lugar de trabajo. A su vez, cuando se les consulta directamente sobre el tema relatan que, generalmente, no humidifican previo al barrido o sacudido, ni tampoco utilizan aspiradora para disminuir la generación de polvo en suspensión durante el aseo, incluso algunos trabajadores refieren no realizar procedimientos de aseo en forma frecuente (esto se aprecia a simple vista en estos lugares de trabajo). A su vez, quienes realizan aseo consideran que, por ser un procedimiento de corta duración 
en relación con la totalidad de la jornada laboral, no reviste una instancia de exposición a pesar del polvo en suspensión que se genera al barrer sin humidificar. El polvo decantado no representa, bajo la perspectiva de los trabajadores, una instancia de exposición a sílice, y por esta razón no consideran que el aseo adecuado sea relevante en relación con el riesgo.

La ausencia de prevencionistas de riesgo y de dispositivos de protección es interpretada por los trabajadores como una señal de escasez o inexistencia de riesgo. Pocos de ellos perciben esta situación como una deficiencia en la gestión del riesgo; bajo su perspectiva, la ausencia de elementos de protección y del profesional respectivo obedece a que el riesgo de exposición es menor o inexistente.

\section{Dimensión presencia de materiales riesgosos}

Los indicadores aludidos en esta dimensión tienen directa relación con las dos anteriores, especialmente con "evidencia de riesgo". El conocimiento de que durante los procesos productivos de su jornada laboral van a tener contacto con polvillo de diverso origen, especialmente de cemento, asbesto, fibrocemento, mármol/ cerámica, aserrín de vidrio, provoca en los trabajadores preocupación por su salud respiratoria, aunque no dan importancia a distinguir el elemento principal o primer responsable de la contaminación del ambiente laboral. Están conscientes de que por la naturaleza de sus oficios siempre tendrán contacto con materiales contaminantes, sin embargo, la necesidad de una fuente laboral, la rutina diaria, y una autopercepción optimista con respecto al riesgo al que están expuestos, entre otros factores, hacen que esta preocupación vaya disminuyendo y pasando a un segundo nivel de importancia, convirtiéndose en algo parecido a la resignación. Basado en los indicadores emanados del análisis de contenidos, se construyeron 13 preguntas. Estas solicitaban al encuestado que, ante una determinada situación, evaluara el riesgo percibido de exposición con una escala de graduación cualitativa de percepción de riesgo de 5 niveles desde sin riesgo hasta muy alto riesgo. Este proceso fue realizado por el equipo de investigadores en base a la proposición, análisis, discusión y evaluación de ítems/preguntas surgidos hasta tener una primera versión del instrumento.

\section{Segunda Etapa: Validación y confiabilidad del instru- mento}

El grupo de expertos al que fue enviado el instrumento concordó positivamente con respecto a la pertinencia de los items. Hubo sugerencias y observaciones en relación con la comprensión de algunos ítems y la sensibilidad de la escala de graduación. En consecuencia, se mejoró la redacción de tres ítems que creaban cierto grado de confusión y se eliminó el nivel de graduación correspondiente a "muy alto riesgo", ya que se estimó que la discriminación entre esta categoría y la de "alto riesgo" resultaría compleja para los trabajadores; a su vez, el objetivo de evaluar niveles progresivos de riesgo se cumple sin la última graduación. La segunda versión del instrumento, producto de las correcciones descritas en el párrafo anterior, fue aplicada a un grupo piloto de 10 trabajadores del rubro de la construcción que realizaban diversas labores con potencial exposición a sílice. Los trabajadores poseían diferentes niveles de educación, desde básica incompleta hasta estudios técnicos, lo cual permitió evaluar integralmente comprensión y desempeño del instrumento. Nuevamente se corrigió la redacción de items que generaban confusión y siguiendo sus sugerencias se simplificó aún más el vocabulario utilizado. A su vez, este proceso fue una instancia para indagar aún más profundamente en la representatividad de las preguntas con respecto al constructo estudiado, no apareciendo aspectos o dimensiones diferentes de las incorporadas hasta el momento y confirmando las existentes. Terminado este proceso, se perfecciona la tercera versión del instrumento.

Para llevar a cabo la validación de constructo, se aplicó la última versión del instrumento a 77 trabajadores de distintos rubros con potencial exposición a sílice (laboratorio dental 37,7\%, marmolería $23,4 \%$, vidriería 36,4\% y construcción 2,6\%). La edad promedio de los participantes fue de 39,3 (DE 12,5 años); 76,6\% eran hombres; $87 \%$ con residencia urbana; $11,7 \%$ con al menos un año de educación básica, 50,6\% con al menos un año de educación media, $\mathrm{y}$ $37,7 \%$ con al menos un año de educación técnica.

Los resultados del AFE muestran que existen tres variables latentes o dimensiones subyacentes entre los ítems medidos y que explican 59,2\% de la varianza producida. El análisis de las correlaciones existentes entre las variables muestra una medida Kaiser Meyer Olkin de 0,82 y una prueba de Barlett significativa ( $\mathrm{p}<$ 0,001 ), lo que establece que existen correlaciones suficientes entre las variables.

El análisis de la matriz de componentes rotados muestra que las preguntas se agrupan en tres dimensiones y se comprueba que concuerdan con el análisis de contenidos previo; sin embargo, la pregunta 8 , incluida en la dimensión dos, muestra una carga factorial baja (0.46), por lo que se decide eliminar (Tabla 1). Este procedimiento aumenta a 61,4\% la varianza explicada por las tres dimensiones.

\section{Tabla 1.}

Matriz de componentes rotados para CuPREOS de 13 preguntas y 3 componentes.

\begin{tabular}{|c|c|c|c|}
\hline \multirow{2}{*}{$\begin{array}{l}\text { En su puesto de trabajo o cercano a éste, qué nivel } \\
\text { de riesgo de exposición a sílice usted percibe cuando: }\end{array}$} & \multicolumn{3}{|c|}{ Componente } \\
\hline & 1 & 2 & 3 \\
\hline 12. No observa elementos de protección personal & 0,857 & 0,024 & 0,172 \\
\hline $\begin{array}{l}\text { 11. En la actividad de trabajo que realiza no se puede } \\
\text { humedecer o agregar agua y se levanta polvo }\end{array}$ & 0,837 & 0,174 & 0,093 \\
\hline $\begin{array}{l}\text { 4. No hay ventilación, es decir, hay espacios cerrados } \\
\text { sin ventanas que hagan circular el aire y sin un } \\
\text { sistema de extracción de polvo }\end{array}$ & 0,792 & 0,221 & 0,010 \\
\hline 1. Hay polvillo visible en el aire & 0,637 & 0,307 & 0,368 \\
\hline $\begin{array}{l}\text { 9. Se asean las áreas de trabajo sin humedecer y } \\
\text { se esparce polvo al ambiente }\end{array}$ & 0,623 & 0,288 & 0,162 \\
\hline $\begin{array}{l}\text { 6. Hay disponibles variados elementos } \\
\text { de protección personal }\end{array}$ & 0,106 & 0,807 & 0,148 \\
\hline $\begin{array}{l}\text { 2. Hay sólo polvillo decantado (el que está encima } \\
\text { de las superficies, máquinas, muebles, etc) }\end{array}$ & 0,123 & 0,697 & 0,011 \\
\hline $\begin{array}{l}\text { 3. En la actividad de trabajo que realiza se agrega } \\
\text { agua o se humedece para disminuir el polvo }\end{array}$ & 0,169 & 0,681 & 0,141 \\
\hline $\begin{array}{l}5 \text { Se asean las áreas de trabajo humedeciendo o } \\
\text { agregando agua y se evita el esparcimiento de } \\
\text { polvo al ambiente }\end{array}$ & 0,207 & 0,576 & 0,089 \\
\hline 8. No hay polvillo visible en el ambiente & 0,304 & 0,456 & 0,317 \\
\hline $\begin{array}{l}\text { 10. Hay espacios abiertos o con gran ventilación } \\
\text { (ventanas abiertas o sistemas de extracción } \\
\text { de polvo permanente) }\end{array}$ & 0,122 & 0,375 & 0,698 \\
\hline $\begin{array}{l}\text { 13. Hay avisos o letreros que informan del } \\
\text { riesgo de exposición a sílice }\end{array}$ & 0,267 & 0,101 & 0,664 \\
\hline $\begin{array}{l}\text { 7. No hay avisos o letreros que informan } \\
\text { del riesgo de exposición a silice }\end{array}$ & 0,518 & 0,394 & 0,55 \\
\hline
\end{tabular}

Método de extracción: análisis de componentes principales. Método de rotación: Varimax con normalización Kaiser. ${ }^{a}$ a La rotación ha convergido en 5 iteraciones. 
Tabla 2.

Matriz de componentes rotados para CuPREOS reducido (9 preguntas y 2 componentes).

\begin{tabular}{|c|c|c|}
\hline \multirow{2}{*}{$\begin{array}{l}\text { En su puesto de trabajo o cercano a éste. qué nivel } \\
\text { de riesgo de exposición a sílice usted percibe cuando: }\end{array}$} & \multicolumn{2}{|c|}{ Componente } \\
\hline & 1 & 2 \\
\hline 12. No observa elementos de protección personal & 0,886 & 0,040 \\
\hline $\begin{array}{l}\text { 11. En la actividad de trabajo que realiza no se puede } \\
\text { humedecer } 0 \text { agregar agua y se levanta polvo }\end{array}$ & 0,837 & 0,151 \\
\hline $\begin{array}{l}\text { 4. No hay ventilación. es decir. hay espacios cerrados sin } \\
\text { ventanas que hagan circular el aire y sin un sistema de } \\
\text { extracción de polvo }\end{array}$ & 0,768 & 0,207 \\
\hline 1. Hay polvillo visible en el aire & 0,706 & 0,326 \\
\hline $\begin{array}{l}\text { 9. Se asean las áreas de trabajo sin humedecer y se } \\
\text { esparce polvo al ambiente }\end{array}$ & 0,662 & 0,305 \\
\hline 6. Hay disponibles variados elementos de protección personal & 0,129 & 0,809 \\
\hline $\begin{array}{l}\text { 3. En la actividad de trabajo que realiza se agrega agua o se } \\
\text { humedece para disminuir el polvo }\end{array}$ & 0,195 & 0,723 \\
\hline $\begin{array}{l}\text { 2. Hay sólo polvillo decantado (el que está encima de las } \\
\text { superficies. máquinas. muebles. etc) }\end{array}$ & 0,132 & 0,696 \\
\hline $\begin{array}{l}\text { 5. Se asean las áreas de trabajo humedeciendo o agregando } \\
\text { agua y se evita el esparcimiento de polvo al ambiente }\end{array}$ & 0,227 & 0,628 \\
\hline
\end{tabular}

Método de extracción: análisis de componentes principales. Método de rotación: Varimax con normalización Kaiser. ${ }^{a}$

a La rotación ha convergido en 3 iteraciones.

Posteriormente, se explora cada dimensión por separado. La primera, llamada "evidencia de riesgo" confirma la reportada en el análisis de contenidos previamente descrito. Esta dimensión abarca 5 preguntas relacionadas con particularidades presentes en los procesos productivos que representan señales de alarma (preguntas 1, 4, 9, 11, 12). El estudio de confiabilidad de esta dimensión arroja un Alfa de Crönbach de 0.85 y una correlación de los items igual o mayor a 0.6. La segunda dimensión, “ausencia de riesgo", quedó conformada por cuatro preguntas que representan circunstancias de mayor seguridad dentro del área laboral y que el trabajador sopesa para contrarrestar las primeras (preguntas 2, 3, 5, 6). La medida de confiabilidad de esta dimensión es de 0,72 , con una correlación de los ítems igual o mayor a 0.45. Se extrajo una tercera dimensión llamada "alerta de riesgo", que se centró en preguntas que aludían a provocar o disminuir señales de alarma, tales como presencia o ausencia de

\section{Figura 1.}

Modelo del análisis factorial confirmatorio de la percepción de riesgo de exposición a sílice.

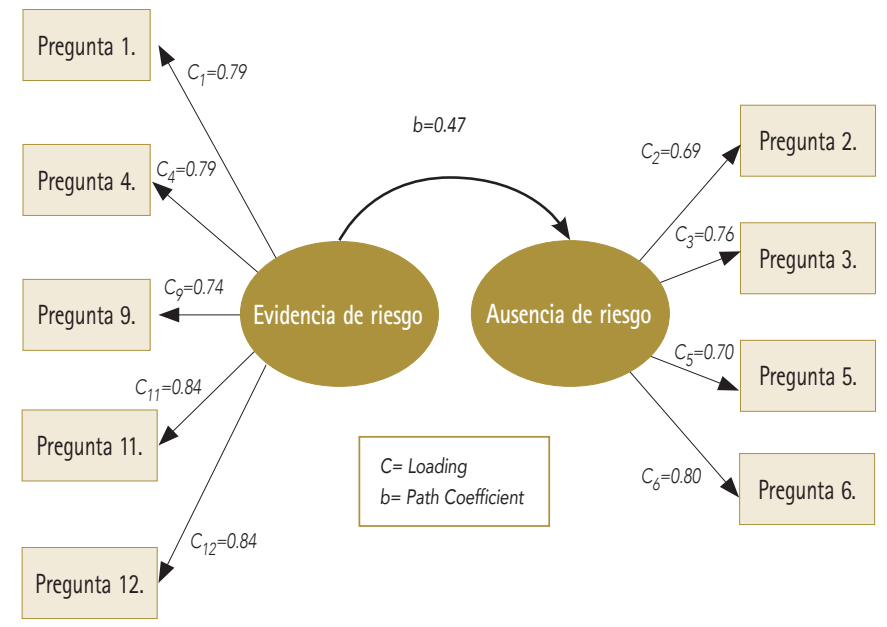

letreros de aviso y ventilación (preguntas 7,10,13). El coeficiente alfa de Crönbach para esta dimensión alcanzó sólo a 0,47 , por lo que se excluyó del instrumento. Se consideró que estas preguntas aportaban poco a la operacionalización del constructo. Por otra parte, el componente materiales peligrosos descrito en el análisis de contenidos, en estricto rigor, quedó representado por preguntas que conformaron la primera dimensión debido a la alta interdependencia de ambos componentes. La varianza explicada por las dos dimensiones que finalmente componen el constructo sólo disminuyó a 60.6\% con la extracción de la tercera dimensión, lo que se consideró despreciable.

Finalmente, el instrumento quedó compuesto por 9 preguntas, con una confiabilidad medida por alfa de Crönbach de 0.84 , conservándose la medida de Kaiser Meyer Olkin $(0,82)$ y una prueba de Barlett significativa ( $p<0.0001)$ (Tabla 2). Estos resultados demuestran una adecuada confiabilidad para el instrumento, basándose en la correlación inter-ítem y en el bajo número de factores que componen el instrumento.31

El análisis confirmatorio a través de GSCA, con el modelo especificado previamente, se muestra en la Figura 1. En el diagrama se describen las variables latentes en las elipses y los items/ preguntas, en los rectángulos. La varianza total explicada por los items/preguntas en el modelo fue de 51\%, los indicadores GFI (Goodness of Fit Index) de 0.991 y SRMR (Standardized Root Mean Square Residual) de 0.080 indican buen ajuste del modelo. Las cargas en los ítems/preguntas de los componentes evidencia de riesgo y ausencia de riesgo tuvieron un rango entre $0.69 \mathrm{y}$ 0.84 ; en cambio, los pesos fueron bajos (0.212 a 0.315$)$, lo que indica que son items/preguntas reflectivas. En el primer componente, $64.1 \%$ de la cantidad promedio de la varianza de los ítems es explicada por su correspondiente variable latente (AVE = 0.641); y en el segundo componente, 54.7\% es explicado por la otra variable latente $(\mathrm{AVE}=0.547)$.

La relación entre ambos componentes se muestra en una sola dirección con un coeficiente de camino (path) significativo ( $\mathrm{p}<$ 0.05). La evidencia de riesgo es un componente identificado como variable latente exógena, mientras que la variable latente ausencia de riesgo es endógena.

\section{DISCUSIÓN}

El presente estudio exploró y analizó cualitativamente aspectos relacionados con la percepción de riesgo de exposición a sílice en trabajadores que por la naturaleza de su ocupación se consideran potencialmente expuestos. Los resultados finales del análisis exploratorio (AFE) y confirmatorio (GSCA) mostraron dos dimensiones subyacentes: evidencia de riesgo y ausencia de riesgo. Un tercer componente, presencia de materiales peligrosos, se correlacionaba altamente en su contenido con el primer componente, por lo que se fusionaron las preguntas en la dimensión evidencia de riesgo. La dimensión alerta de riesgo obtuvo un bajo coeficiente de Crönbach, por lo que se excluyó, permitiendo un mejor comportamiento y un buen ajuste del modelo. De las 2 dimensiones que permanecieron se desprendieron 9 items/preguntas que conformaron el Cuestionario de Percepción de Riesgo de Exposición Ocupacional a Sílice (CuPREOS). La consistencia interna, estratificada por dominio (Alfa de Crönbach de 0.85 para evidencia de riesgo y 0.71 para ausencia de riesgo), demuestra que los ítems/preguntas de cada componente están correlacio- 
nados entre sí, lo que indica que son adecuados para medir ambas dimensiones como componentes del constructo percepción de riesgo de exposición a sílice. ${ }^{37,38}$ La dimensión evidencia de riesgo se manifiesta como una variable exógena ya que no es influida por el componente ausencia de riesgo, sino por el contrario, esta última es dependiente de la primera (endógena). Esto se manifiesta en los relatos de los trabajadores que perciben el riesgo sopesando, en primer lugar, los factores que les indican algún nivel de riesgo para su integridad física y posteriormente analizan aquellos factores que pudieran contrarrestar este efecto; sin embargo, prima la primera percepción.

Con respecto a las limitaciones de este estudio, es necesario mencionar que la muestra de trabajadores entrevistados para el proceso de validación de constructo no fue escogida probabilísticamente, sino que provenía de empresas del área urbana cuyo rubro se considera potencialmente expuesto a sílice, quedando sujetos al consentimiento de las empresas de dejar participar a sus trabajadores. El número de trabajadores encuestados fue menor a lo planificado inicialmente, sin embrago, el tamaño de la muestra cumple con los criterios mínimos necesarios para este tipo de evaluación. Por otra parte, las características sociodemográficas y condiciones de trabajo de aquellos que no fueron encuestados son similares a las de los trabajadores de la muestra, ya que pertenecen a las mismas empresas o eran de empresas de características similares. De todos modos, la aplicación del cuestionario a otras poblaciones con similares características puede aumentar la evidencia de confirmación de las propiedades psicométricas desarrolladas en este trabajo, así como también la estabilidad de su estructura.

La percepción de riesgo es un constructo que implica complejidades sociales y culturales importantes, sumado a ello, la diversidad entre los trabajadores potencialmente expuestos a sílice, en relación con su nivel educacional y cultural, no permiten aplicar instrumentos complejos. Los autores de este trabajo consideran que un instrumento como este, que atrapa los componentes centrales del constructo, y que considera solo preguntas esenciales, de estructura sencilla, es efectivo para la medición del constructo y acerca objetivamente al investigador a la realidad que se estudia.

No habiéndose encontrado en la literatura otro instrumento similar, se considera que la aproximación a la percepción de riesgo de exposición a sílice en trabajadores potencialmente expuestos a través de este instrumento es un aporte importante para evaluar y planificar estrategias preventivas con los trabajadores. Existe suficiente evidencia que sugiere estudios de percepción de riesgo e intervenciones en este aspecto para lograr mejorar resultados en la prevención de daños a la salud, especialmente para el aumento del involucramiento de los trabajadores en la gestión del riesgo en el área ocupacional. ${ }^{39-41}$

Es de especial consideración lo que menciona Dake en su revisión de literatura ${ }^{16}$, con respecto a que la percepción de riesgo es socialmente construida y culturalmente sesgada en el sentido que los individuos influyen y pueden rediseñar las opiniones prevalentes en sus propios círculos sociales. Este es, probablemente, el mejor argumento para estudiar y evaluar la percepción de riesgo en grupos en los cuales es necesario intervenir para mejorar conductas y hábitos preventivos en forma más eficiente.

\section{Agradecimientos}

A la Secretaría Regional Ministerial de Salud de La Araucanía, Chile, quien a través de la Unidad de Salud Ocupacional, específicamente Sr. Juan Fernando Urrutia, nos brindó asesoría técnica especializada y colaboración en la recolección de datos.

A la Universidad de La Frontera, Temuco, Chile, quien a través de la Dirección de Investigación aportó con los fondos necesarios para la realización de este estudio: Proyecto DIUFR0 DI12-0017. 


\section{REFERÊNCIAS}

1. American Thoracic Society. Adverse effects of crystalline silica exposure. Am J Resp Crit Care. 1997;155(2):761-765.

2. Calvert GM, Rice FL, Boiano JM, Sheehy JW, Sanderson WT. Occupational silica exposure and risk of various diseases: an analysis using death certificates from 27 states of the United States. Occup Environ Med. 2003;60(2):122-129.

3. Rice F. International Programme of Chemical Safety: Crystalline Silica, Quartz [on line]. Geneva: World Health Organization; 2000 [cited 2017 dec 27]. (CICAD; 24). Available from: http://www.inchem.org/documents/cicads/cicads/ cicad24.htm

4. Bernales B, Alcaino J, Solis R. Situación de exposición laboral a sílice en Chile. Cienc Trab [en línea]. 2008 [citado may 2018]; 10(27):1-6. Disponible en: http:// www.ispch.cl/sites/default/files/Silice_en_chile.pdf

5. Aggarwal BD. Lactate dehydrogenase as a biomarker for silica exposureinduced toxicity in agate workers. Occup Environ Med. 2014;71(8):578-582.

6. Birk T, Guldner K, Mundt KA, Dahmann D, Adams RC, Parsons W. Quantitative crystalline silica exposure assessment for a historical cohort epidemiologic study in the German porcelain industry. J Occup Environ Hyg. 2010;7(9):516528.

7. Sivanmani $K$, Rajathinakar V. Silicosis in Coimbatore district of Tamil Nadu: A passive surveillance study. Indian J Occup Environ Med. 2013;17(1):25-28.

8. McCool JP, Reeder Al, Robinson EM, Petrie KJ, Gorman DF. Outdoor workers' perceptions of the risks of excess sun-exposure. J Occup Health. 2009;:51(5):404411.

9. Damalas CA, Hashemi SM. Pesticide risk perception and use of personal protective. Agrociencia [on line]. 2010 [cited 2017 Oct 30]; 44:363-371. Available from: http://www.scielo.org.mx/pdf/agro/v44n3/v44n3a10.pdf

10. Valim MD, Marziale MH, Richart-Martinez M, Sanjuan-Quiles A. Instruments for evaluating compliance with infection control practices and factors that affect it: an integrative review. J Clin Nurs. 2014; 23(11-12):1502-1519.

11. Bard D, Burdett G. Exposure of UK Industrial Plumbers to Asbestos, Part II : Awareness and Responses of Plumbers to Working with Asbestos During a Survey in Parallel with Personal Sampling. Ann Occup Hyg. 2007; 51(2):113119.

12. Arezes PM, Miguel AS. Risk perception and safety behaviour: A study in an occupational environment. Safety Sci. 2008; 46(6):900-907.

13. Caponecchia C, Sheils I. Perceptions of personal vulnerability to workplace hazards in the Australian construction industry. J Safety Res. 2011; 42(4):253-258.

14. Sharot T. The Optimism Bias. Curr Biol [on line]. 2011 [cited 2018 Jan 9]; 21(23):R941-945. Available from: http://www.cell.com/article/S0960982211011 912/fulltext

15. Garrett N, Sharot T. How robust is the optimistic update bias for estimating self-risk and population base rates? PloS ONE [on line]. 2014 [cited 2017 Dec 7]; 9(6):e98848. Available from: http://www.pubmedcentral.nih.gov/articlerender.fcgi?artid $=4051586 \&$ tool $=$ pmcentrez\&rendertype $=$ abstract

16. Dake K. Myths of Nature: Culture and the Social Construction of Risk. J Soc Issues. 1992;48(4):21-37.

17. Brewer NT, Weinstein ND, Cuite CL, Herrington JE. Risk perceptions and their relation to risk behavior. Ann Behav Med. 2004; 27(2):125-130.

18. Vernon SW. Risk perception and risk communication for cancer screening behaviors: a review. Monogr J Natl Cancer Inst. 1999; 25:101-119.

19. Stewart-Taylor AJ, Cherrie JW. Does risk perception affect behaviour and exposure? A pilot study amongst asbestos workers. Ann Occup Hyg. 1998; 42(8):565-569.

20. Arezes PM, Miguel AS. Hearing protection use in industry: The role of risk perception. Safety Sci. 2005; 43(4):253-267.

21. Si S, Carey RN, Reid A, Driscoll T, Glass DC, Peters $S$ et al. The australian work exposures study: prevalence of occupational exposure to respirable crystalline silica. Ann. Occup. Hyg. 2016; 60(5):631-637.
22, Kirkeskov L, Hanskov DJ, Brauer C. Total and respirable dust exposures among carpenters and demolition workers during indoor work in Denmark. J Occup Med Toxicol [on line]. 2016 [cited 2018 jan 25]; 11:45. Available from: https:// www.ncbi.nlm.nih.gov/pmc/articles/PMC5028992/pdf/12995_2016_ Article_134.pdf

23. Scarselli A, Corfiati M, Marzio DD, lavicoli S. Evaluation of workplace exposure to respirable crystalline silica in Italy. Int J Occup Environ Health. 2014; 20(4):301-307.

24. Radnoff D, Todor MS, Beach J. Occupational Exposure to Crystalline Silica at Alberta Work Sites. J Occup Environ Hyg. 2014; 11(9):557-570.

25. Pérez-Alonso A, Córdoba-Doña JA, Millares-Lorenzo JL, Figueroa-Murillo $E_{\text {, }}$ García-Vadillo C, Romero-Morillo J. Outbreak of silicosis in Spanish quartz conglomerate workers. Int J Occup Environ Health. 2014; 20(1):26-32.

26. Martínez-González C. Cambio en el perfil de las enfermedades por exposición a la inhalación de silice. Arch Bronconeumol. 2018; 54(1):5-6.

27. Benach J, Solar O, Vergara M, Vanroelen C, Santana V, Castedo A et al. Six Employment Conditions and Health Inequalities: A Descriptive Overview. Int J Health Serv. 2010; 40(2):269-280.

28. Bernales B, Marchetti N, Jaramillo H. Plan Nacional para la erradicación de la silicosis. Estrategia 2009-2030 [on line]. Santiago de Chile: Ministerio de SaludMinisterio del Trabajo y Previsión Social; 2009 [citado 20 dic 2017]. 39 p. Disponible en: http://web.minsal.cl/portal/url/item/7d35c3dc76610f33e04001 011e015333.pdf.

29. Gobierno de Chile. Ministerio de Economía. Emprendimiento formal e informal en Chile; Análisis a partir de los resultados de la Tercera Encuesta de microemprendimiento 2013 [en línea]. Santiago: Gobierno de Chile; 2013 [citado ene 2018].19 p. Disponible en: http://www.innovacion.cl/wp-content/uploads/ 2013/12/Boletin-EME-3-Formalidad1.pdf

30. Carlson ME, Clark FA. The Search for Useful Methodologies in Occupational Science. Am J Occup Ther. 1991;45(3):235-241.

31. Oliver J, Benet-Martinez V. Measurement: Reliability, construct validation, and scale construction. In: Reis HT, Judd CM, editors. Handbook of research methods in social and personality psychology. Cambridge: Cambridge University Press; 2000. p. 339-369..

32. Hsieh HF, Shannon SE. Three approaches to qualitative content analysis. Qual Health Res. 2005;15(9):1277-1288.

33. Pesudovs K, Burr JM, Harley C, Elliott DB. The development, assessment, and selection of questionnaires. Optom Vis Sci. 2007; 84(8):663-674.

34. Williams B, Brown T, Onsman A. Exploratory factor analysis: A five-step guide for novices. Australas J Paramed [on line]. 2012 [cited 2018 mar 2]; 8(3):1-13. Available from: https://ajp.paramedics.org/index.php/ajp/article/view/93/90.

35. Henson RK. Use of exploratory factor analysis in published research: common errors and some comment on improved practice. Educ Psychol Meas. 2006; 66(3):393-416.

36. Hogarty KY. The quality of factor solutions in exploratory factor analysis: the influence of sample size, communality, and overdetermination. Educ Psychol Meas. 2005;65(2):202-226.

37. Bland JM, Altman DG . Statistics notes: Cronbach's alpha. BMJ [on line] 1997 [cited 2018 Jan 5]; 314:572. Available from: http://www.bmj.com/ content/314/7080/572.full.pdf+html

38. Tavakol M, Dennick R. Making sense of Cronbach's alpha. Int J Med Educ. 2011; 2:53-55.

39. Xia N, Wang X, Griffin MA, Wu C, Liu B. Do we see how they perceive risk? An integrated analysis of risk perception and its effect on workplace safety behavior. Accid Anal Prev. 2017; 106:234-242.

40. Sullivan-Wiley KA, Short Gianotti AG. Risk Perception in a Multi-Hazard Environment. World Dev. 2017; 97:138-152.

41. Taylor WD, Snyder LA. The influence of risk perception on safety: A laboratory study. Safety Sci. 2017; 95:116-124. 